\title{
TRATAMIENTO DE UN CASO CLÍNICO DE INTESTINO IRRITABLE
}

\author{
Ana M" Regueiro Ávila \\ Luis Valero Aguayo
}

Servicio de Atención Psicológica. Facultad de Psicología. Universidad de Málaga

\section{RESUMEN}

Se describe un caso clínico con un problema de "intestino irritable", varón de 19 años con una historia de 3 años de problemas gastrointestinales. Se ha aplicado un tratamiento en 20 sesiones, tras un análisis funcional adaptado a sus caracteristicas individuales. Incluyó una fase educativa sobre esta problemática, y un conjunto de técnicas cognitivoconductuales que incluian: contrato de contingencias, control estimular sobre comidas y pautas alimenticias, control del estrés $y$ emociones, $y$ reestructuración cognitiva. Se ha utilizado un diseño de caso único, con línea base concurrente en los episodios de dolor, y varias sesiones de seguimiento hasta un año. Los resultados mostraron una disminución rápida de los problemas en las cinco primeras semanas, hasta su eliminación casi completa al final del tratamiento.

Palabras clave: INTESTINO IRRITABLE, tRATAMIENTO MULTICOMPONENTES, ANALISIS FUNCIONAL, CONTRATO DE CONTNGENCIAS. 


\section{SUMMARY}

A clinical case with a problem of irritable bowel is described. He was 19 year-old, male with a 3 year-old history of that problem. A functional analysis adapted to its individual characteristics was used, and a treatment multiple was applied in 20 sessions. The treatment had an educational phase on this problem, and a group of behavioral-cognitive techniques (contingency contract, stimuli control about foods and nutritious rules, stress and emotional control, and restructuring thoughts). An only-case design was used, with three base-lines about the pain episodes. The following phase was during a year. The results showed a quick decrease of the problems over five weeks, and the generalization was complete through that person's daily life.

Key words: IRRITABLE BOWEL, MULTICOMPONENT TREATMENT, FUNCTONAL ANALYSIS, CONTNGENCY CONTRACT.

\section{INTRODUCCIÓN}

El denominado "síndrome de intestino irritable" es un trastorno funcional, puesto que no existen causas orgánicas detectadas al respecto, que se caracteriza por diversas alteraciones intestinales como dolor abdominal, diarrea, estreñimiento, y tarnbién otras características asociadas como fatiga, insomnio y cefaleas. Este diagnóstico suele darse por exclusión, es decir cuando aparece esa sintomatología sin un trastorno fisiológico como causa, cuando el individuo toma medicación sin resultado, y además este malestar altere sus condiciones de vida (Thompson, 1984). Existen razones biológicas que explican el origen del trastorno, tales como una mayor responsividad en el colon que daría lugar al estreñimiento y dolor, y una menor tolerancia a la distensión de esa musculatura, que daría lugar a diarreas. Pero también se ha argumentado que se trata del aprendizaje de una conducta inadaptada de enfermedad crónica (Fernández, 1989), puesto que existen hábitos alimentarios y digestivos característicos en estas personas, y además los problemas asociados indican un fuerte factor desencadenante en situaciones de estrés y 
ansiedad. También se ha señalado que son personas con unas características definidas respecto a la enfermedad, que Whitehead et al. (1982) denominan "comportamiento aprendido de enfermedad crónica" (conversaciones exclusivas sobre enfermedad, toma continua de medicación, visitas a distintos especialistas, incapacitación en la vida diaria y cronificación del problema). Por tanto el tratamiento psicológico iría encaminado al aprendizaje de conductas saludables, de bienestar, y tratar de modificar hábitos de vida y conductas desadaptadas relacionadas con la salud. Al ser un trastomo crónico y que origina múltiples consultas médicas, el mismo problema se ve agravado por las contingencias de reforzamiento familiar y profesional. De esta forma, el ambiente social cronifica una patología al no encontrar causas fisiológicas y dar mucha importancia a todas las quejas variadas y problemas continuos que suelen presentar estas personas.

Existen estudios controlados que muestran la eficacia diferencial de un tratamiento psicológico frente al médico en este tipo de casos crónicos (Guthrie et al, 1992; Heymann et al., 2000), y la eficacia de un tratamiento multicomponentes frente a otros tratamientos no conductuales o placebo (Blanchard et al., 1992; Greene y Blanchard, 1994; Payne y Blanchard, 1995). Aunque también se ha criticado la falta de tratamientos empíricamente validados para este tipo de trastornos, incluyendo todo tipo de tratamientos médicos, dietéticos, farmacológicos, psicológicos o alternativos (Fennerty, 2003).

Los tratamientos conductuales sobre este trastomo se iniciaron con Latimer (1983) y Whitehead (1985), por un lado, con el manejo de contingencias en su ambiente cotidiano, y por otro, con la reducción del estrés mediante relajación, biofeedback, desensibilización y técnicas de afrontamiento. En castellano, Femández (1989) presenta un estudio comparativo sobre 40 pacientes con sindrome de intestino irritable, aplicando a un grupo tratamiento médico convencional, a otro grupo tratamiento de manejo de contingencias, y un tercero de afrontamiento del estrés. Estos dos últimos grupos redujeron significativamente los síntomas intestinales y las quejas respecto al grupo médico de control, pero comparativamente los mejores resultados se obtuvieron en el grupo de manejo de contingencias, tanto en los síntomas gastrointestinales como en los demás síntomas no digestivos. Y también González, García y Fernández (1992) muestran 
dos casos clínicos diferentes donde aplicaron un tratamiento de manejo de contingencias para uno de ellos, mediante la búsqueda de actividades y relaciones sociales alternativas, la extinción de conversaciones sobre enfermedad y el reforzamiento social del marido. Mientras que en el otro caso aplicaron un programa de manejo del estrés, mediante relajación progresiva, manejo de habilidades de afrontamiento de situaciones desencadenantes, y control de pensamientos antecedentes a esas situaciones. Ambos casos tuvieron buenos resultados en un seguimiento de tres meses.

Se presenta aqui un caso clínico donde la evaluación inicial y el análisis funcional determinaron múltiples factores de mantenimiento de esta problemática de intestino irritable, por lo que se realizó un tratamiento múltiple encaminado a solventar cada uno de esos factores. De esta forma se presenta una casuística algo más compleja, donde el análisis funcional resulta fundamental para adecuar las características de un tratamiento individualizado. Por otra parte, para mostrar la eficacia del tratamiento, se ha realizado un diseño de caso único con varias líneas bases simultáneas de la sintomatología de dolor, y el seguimiento de un año.

\section{DESCRIPCIÓN DEL CASO}

Juan era un estudiante varón de 19 años, que acudió a consulta del Servicio de Atención Psicológica porque decía padecer una enfermedad psicosomática que le afectaba a su estado de ánimo y su autoestima, le daba muchas vueltas a las cosas, no terminaba lo que emprendía y manifestaba cierta agresividad. A partir de las entrevistas iniciales se extrajo la siguiente información: padecía un trastorno de intestino irritable desde hacía unos tres años, aunque había comenzado antes, empeoró a partir de una pelea con su mejor amigo. Había pasado por varios especialistas (psiquiatras, digestivos, radiólogos, estomatólogos, etc.), con numerosas pruebas y análisis, que habían descartado un origen orgánico de su problema, y sólo indicaban que la mucosa del estómago estaba algo irntada. Los diagnósticos de los psiquiatras señalaban la presencia de rasgos obsesivos de personalidad, pensamientos circulares y somatizaciones, además de síntomas depresivos y baja autoestima. No habían dado el diagnóstico específico 
de "síndrome de intestino irritable", pues algunas de sus características, según los criterios de Manning et al. (1978), no aparecian completas en esta persona. Estos criterios incluyen: (1) dolor abdominal que se alivia con la defecación, (2) deposiciones frecuentes, (3) deposiciones poco consistentes o diarrea, (4) eliminación de moco, (5) sensación de evacuación incompleta, y (6) distensión abdominal.

En el momento de la evaluación estaba tomando tres tipos de tranquilizantes y un protector del estómago. La característica principal es que tenia episodios diarios de dolor, que comenzaban dos o tres horas después de haber comido, entonces comía de nuevo y se le quitaban, pero al tiempo volvian a aparecer. Esos episodios de dolor cursaban también con gases, ruidos estomacales, quemazón, diarreas y dolor agudo en el estómago. También tenía una frecuencia muy alta de micciones diurnas, aproximadamente 20 al día, no retenía mucho la orina porque le dolía, y tampoco durante la noche, pues se levantaba varias veces para ir al baño. No se habían detectado causas orgánicas para esos trastornos.

Juan habia adoptado determinados patrones alimentarios, con una dieta autoimpuesta, puesto que -según él- habia muchos alimentos que no le sentaban bien. Asi evitaba las legumbres, verduras, chocolate, tomate, alcohol, bebidas gaseosas, y algunas otras. Además tenía unos hábitos de comida muy rígidos, de comidas rápidas, precocinadas, y comiendo siempre muy deprisa. Cuando tenía dolores de estómago comía algo para que se le quitara, y a las pocas horas tenía otra vez esos episodios de dolor.

Estos problemas físicos le estaban afectando a nivel personal, pues siempre se encontraba nervioso $\theta$ irascible. Le daba continuamente vueltas a su problema, no se podía concentrar, y su rendimiento académico había bajado. Cada vez que experimentaba ese dolor dejaba lo que estaba haciendo, delegaba responsabilidades, se aislaba de los amigos o discutía con ellos. Siempre se encontraba cansado, con cambios continuos de humor. Estos cambios también estaban afectando negativamente a sus relaciones sociales (que eran escasas) y familiares, pues las discusiones con sus padres eran muy frecuentes.

Por otro lado, también aparecieron una serie de pensamientos continuos sobre su orientación sexual, pues nunca había tenido novia, 
y aunque se había sentido atraído por una chica, también se fijaba en los chicos. Había tenido juegos sexuales con chicos en la infancia, y ahora tenía una gran ansiedad y preocupación sobre cuál era su orientación sexual. En el historial de personas con sindrome de intestino irritable es frecuente que aparezcan episodios de abuso sexual en mayor o menor grado, y que aparezcan también problemas depresivos, pensamientos negativos sobre sí mismo y baja autoestima, en la elaboración de esas historias personales (Ilnycky y Bernstein, 2002; Rossouw, Stuart y Pretorius, 2003; Walker et al., 1993).

\section{MÉTODO}

\section{Evaluación}

Se realizaron 4 sesiones de evaluación en forma de entrevistas, donde se utilizó también una entrevista conductual centrada en el problema y una entrevista semiestructurada del propio Servicio de Atención Psicológica. También se aplicó el cuestionario de personalidad 16PF-5 (Rusell y Karol, 1995) para detectar otros posibles problemas psicopatológicos. Se recogió también la información médica y farmacología actual. Se le pidieron autorregistros diarios sobre las características de su alimentación (tipo y cantidad de comida, lugares, forma y tiempo empleado), y sobre los episodios de dolor (situaciones, pensamientos antecedentes, sintomas físicos, intensidad subjetiva del dolor en una escala $0-10$, qué hacia para resolverlos, posibles consecuencias, y duración del episodio). También se elaboró una escala de valoración pre-post para todo el conjunto de características y síntomas que aparecian. Todos los ítems se valoraban en un grado de 0 a 4 según la frecuencia de aparición (0-ninguna vez en el último mes, 1-alguna vez en las últimas semanas, 2-dos o tres veces a la semana, 3-casi todos los dias de la semana, 4-varias veces al día). En el Anexo 1 aparece la relación de ítems de esta escala.

En cuanto a sus hábitos alimentarios, obtenidos a partir de los autoregistros de una semana, aparecen desayunos rápidos (leche y tostadas), toma entre horas sándwiches y bebidas sin gas, también galletas, donuts, etc., para prevenir los dolores de estómago. A mediodia almuerza delante de la tele él solo, también muy rápido (sobre 
10-15 minutos), aunque es la comida habitual de la familia. Por la noche suele cenar fuera (pizza, camperos, bocadillos), y cuando to hace en casa también es muy rápido (15 minutos). En la Figura 1 aparece la línea base sobre la frecuencia, intensidad y duración de los episodios de dolor, obtenidos durante dos semanas. Aparecen numerosos episodios de dolor y molestias gástricas (movimientos intestinales, ardor, ganas de vomitar, etc.) que suelen durar entre $\mathbf{3 0}$ minutos y una hora. Casi todos ellos aparecen relacionados con situaciones de ansiedad, disgustos o discusiones, o anticipadamente a situaciones estresantes.

Figura 1.- Esquema de la hipótesis de análisis funcional de mantenimiento del problema del caso clínico

\begin{tabular}{|c|c|c|}
\hline $\begin{array}{l}\text { ESTIMULACIÓN } \\
\text { ANTECEDENTE }\end{array}$ & RESPUESTAS & $\begin{array}{l}\text { ESTIMULACIÓN } \\
\text { CONSECUENTE }\end{array}$ \\
\hline $\begin{array}{l}\text { Exámenes } \\
\text { Viajes } \\
\text { Cambios de rutina } \\
\text { Conflictos familiares } \\
\text { Preocupaciones } \\
\text { Situación de comida }\end{array}$ & $\begin{array}{l}\text { FISIOLÓGICAS } \\
\text { Dolor abdominal } \\
\text { Ardores } \\
\text { Ansias } \\
\text { Retortijones } \\
\text { Gases } \\
\text { Urgencia urinaria }\end{array}$ & $\begin{array}{l}\text { Ref. NEGATIVO } \\
\text { Toma comida } \\
\text { Toma medicación } \\
\text { Se acuesta o relaja } \\
\text { Va al WC }\end{array}$ \\
\hline $\begin{array}{l}\text { Movimientos intestinales } \\
\text { Dolor }\end{array}$ & $\begin{array}{l}\text { MOTORAS } \\
\text { Agitación constante } \\
\text { Se va de donde esté } \\
\text { Comunica sus dolores }\end{array}$ & $\begin{array}{l}\text { Ref. POSITIVo } \\
\text { Atención de familiares } \\
\text { Atención de amigos }\end{array}$ \\
\hline $\begin{array}{l}\text { Movimientos intestinales } \\
\text { Preocupaciones } \\
\text { Anticipación de } \\
\text { situaciones }\end{array}$ & \begin{tabular}{l}
\multicolumn{1}{c}{ COGNITIVAS } \\
Pensamientos negativos \\
sobre su estado \\
Hipervigilancia de los \\
sintomas \\
Hipersensibilidad ante los \\
demás
\end{tabular} & $\begin{array}{l}\text { Autorreforzamiento } \\
\text { Confirmación de } \\
\text { pensamientos } \\
\text { anticipatorios } \\
\text { Pensamientos de falta de } \\
\text { control }\end{array}$ \\
\hline
\end{tabular}

\section{Análisis funcional}

Podría hipotetizarse como variables de adquisición de esta problemática, una cierta predisposición biológica (siempre había tenido 
molestias y cierta hipersensibilidad, con baja tolerancia al dolor), unido a unas pautas de alimentación inadecuadas (ingestión muy rápida, de pie, con poca masticación, ingestión de liquidos durante la comida, etc.), que ante un contexto y episodios estresantes (vivir con la abuela, pelea con el amigo, preocupaciones sobre sexualidad y pensamientos perfeccionistas) desencadenaría ese problema de dolor crónico abdominal de carácter funcional. Probablemente condicionados ante situaciones y pensamientos de tipo aversivo. Por otra parte, estos episodios estarían al principio reforzados por la preocupación familiar sobre su salud, los cuidados maternos y las continuas consultas médicas; a lo que se añadiría el reforzamiento negativo que obtenían esos episodios al delegar responsabilidades y utilizarlas como excusa para otras tareas.

Como posible análisis funcional sobre el mantenimiento actual de esa problemática, destacaba una serie de situaciones desencadenantes características de estrés (exámenes, viajes, cambios de rutina, conflictos familiares, preocupaciones), además de pensamientos anticipatorios sobre el propio dolor y las situaciones desencadenantes. Como respuestas fisiológicas aparecian todos esos episodios de trastornos estomacales, además de sudoración, presión urinaria y activación en general; al tiempo que como respuestas cognitivas tenía pensamientos distorsionados y negativos del tipo: "no aguanto mas... no voy a poder salir de esto... otra vez lo tengo... ya me esta dando... esto no se me va a quitar nunca...". Este tipo de respuestas se veía reforzada negativamente (disminuía o eliminaba el dolor) cuando comía algo, se acostaba o relajaba tranquilo en casa, delegaba y evitaba tareas que debía realizar, y evitaba también situaciones o compromisos sociales. $Y$ al mismo tiempo, también era reforzada positivamente por la atención de sus familiares y allegados; incluso era autorreforzada por sus pensamientos confirmatorios sobre el dolor, sobre el carácter crónico de sus problemas y la imposibilidad de llevar una vida normal (ver Figura 2).

Según este posible análisis funcional, se propusieron como pautas de actuación: (1) eliminar el reforzamiento positivo y negativo de esos episodios, mediante asunción de responsabilidades, eliminación de la atención familiar, (no comunicar episodios), desvío del foco de atención sobre los síntomas físicos, y aprendizaje de respuestas alternativas 
Figura 2.- Frecuencia, intensidad y duración total de los episodios durante la Inea base, tratamiento y seguimiento
Linea-Base
Tratamiento
Seguimiento
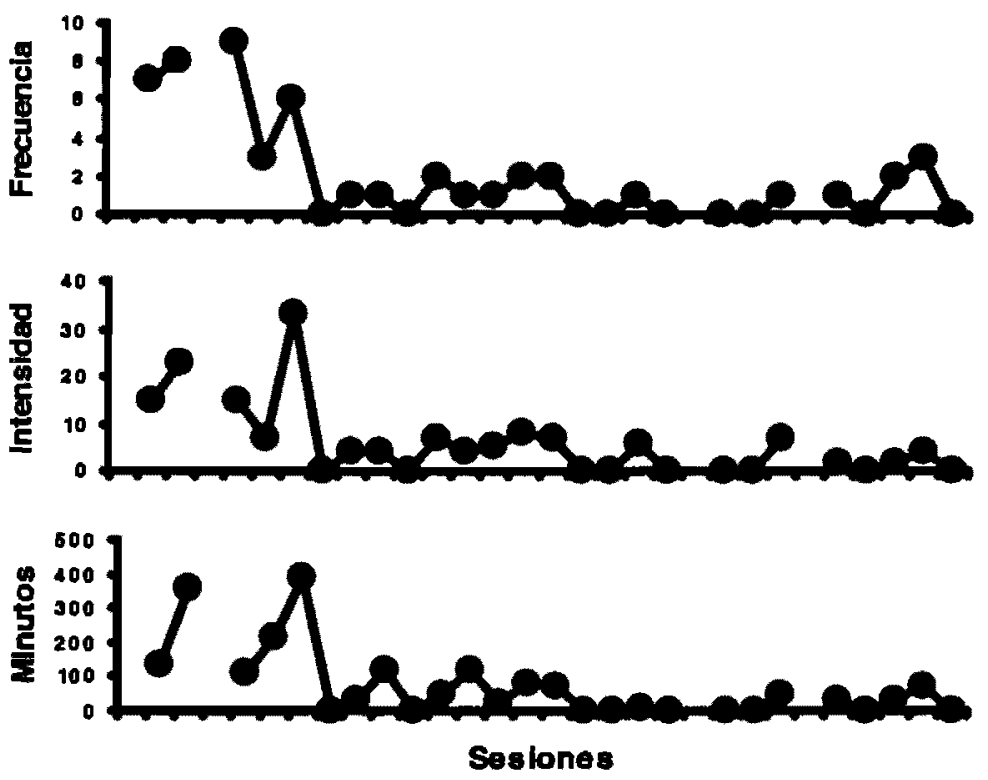

ante situaciones sociales y conflictivas; (2) contracondicionar sus respuestas de estrés mediante relajación y respiración, al tiempo que se intentaban cambiar sus pautas alimentarias para reducir los trastornos digestivos; (3) afrontar con otro estilo cognitivo las situaciones de estrés, sin pensamientos anticipatorios, eliminando progresivamente preocupaciones y pensamientos distorsionados sobre sí mismo y su sexualidad.

\section{Tratamiento}

Estas pautas de tratamiento se llevaron a cabo progresivamente en un total de 20 sesiones (semanales y otras quincenales en función de la disponibilidad). La primera fase (2 sesiones) consistio en tareas educativas de información sobre el problema de intestino irritable, los factores desencadenantes, pensamientos anticipatorios y formas de 
afrontarlo. Aquí se incluyeron varios artículos para su lectura como biblioterapia, que fueron comentados en la siguiente sesión.

En una segunda fase (sesiones 3 a 10) se inició un contrato de contingencias por la realización de conductas incompatibles, con objeto de corregir sus hábitos de alimentación, y disminuir el reforzamiento social de sus "quejas psicosomáticas". Entre esos cambios se incluyó el control estimular durante la comida, control postural, sin TV, comer con el resto de familia y no levantarse de la mesa hasta terminar todos, tiempo de masticación, bocados pequeños, y aumentar la ingesta de líquidos. $Y$ al mismo tiempo no comunicar sus quejas y no evitar situaciones familiares en las que utilizaba sus dolores como excusa. A lo largo de estas sesiones se introdujeron también algunas lecturas sobre percepción del dolor, errores cognitivos, problemas sexuales y orientación sexual, que servian de base para conversaciones, discusión socrática y generación de ideas alternativas sobre su problema.

En una tercera fase (sesiones 11 a 16) se introdujo un proceso progresivo de exposición ante diferentes situaciones estresantes que antes evitaba (ir a clase, estar sin comer, determinados alimentos, tareas en casa, etc. $Y$, por otra parte, la respiración abdominal y la relajación progresiva para disminuir la ansiedad fisiológica ante situaciones problemáticas. Como técnica de autocontrol emocional se utilizó también "tiempo fuera" como forma de eliminar sus emociones y reacciones de enfado o ira ante discusiones familiares y con otras personas.

En una cuarta fase (sesiones 17 a 20) se realizó un entrenamiento en resolución de problemas para afrontar de otra forma esas situaciones conflictivas, además de prevención de recaídas y generalización de esas habilidades. En estas últimas sesiones siempre se hacía un repaso de las diversas técnicas, de los cambios producidos y las estrategias ya utilizadas en su vida diaria. Finalmente se le incluyó en el grupo de aprendizaje de habilidades sociales del mismo Servicio de Atención Psicológica, para completar el tratamiento.

Se realizaron 5 sesiones de seguimiento, en diversos meses, hasta obtener un seguimiento final un año después de terminar la terapia. En estas sesiones se revisaban los registros, problemas ocurridos durante ese tiempo y los cambios producidos. 


\section{RESULTADOS}

Tras las primeras sesiones de tratamiento, los episodios disminuyen en intensidad, frecuencia y duración (ver Figura 1). Empieza a identificar los desencadenantes arnbientales de sus problemas, las situaciones estresantes, y los pensamientos anticipatorios. Se empiezan a producir cambios en su dieta y forma de alimentación, hay un aumento de su satisfacción y sensación de control sobre su problema. Aparecen ya semanas completas sin dolor. Desde la sexta sesión comienza por su cuenta a realizar ejercicio físico, retoma sus estudios y empieza a ir a algunas clases. A partir de la $11^{\mathrm{a}}$ sesión empieza a autoexponerse a situaciones antes evitadas, $y$ aparecen varias semanas completas sin dolor alguno. En el periodo de vacaciones apenas aparecen 2 episodios de dolor en casi dos meses.

Al terminar el tratamiento no aparecen episodios de dolor, asiste a clases regularmente y aprueba los exámenes a los que se presenta. Su alimentación está normalizada. No toma medicación alguna. Juan es perfectamente capaz de reconocer los sucesos, emociones o pensamiento que le estresan, y es capaz de pararse y conceptualizarlos de otra forma, tiene capacidad de autocontrol y resolución de problemas que se le presentan. También ha ampliado el círculo de sus amistades y actividades de ocio cotidianas.

La evaluación pre-post con el cuestionario específico (ver Anexo 1) muestra una diferencia de 71 puntos inicialmente, frente a 9 puntos al terminar el tratamiento. En la Figura 2 puede observarse el cambio progresivo en los episodios de dolor a lo largo de todo el proceso. Al año de seguimiento se mantienen estos resultados, han aparecido algunos episodios de dolor muy esporádicos, pero con poca intensidad y duración, que pueden considerarse normales dado el extenso periodo de tiempo que comprenden.

\section{CONCLUSIONES}

Se ha mostrado la evaluación y tratamiento de un caso de "síndrome de intestino irritable", donde la hipótesis del análisis funcional marca las pautas de actuación. De esta forma se ha mostrado cómo en un problema complejo, con múltiples variables de mantenimiento, es 
posible desarrollar un tratamiento individualizado, por etapas, con objetivos terapéuticos para cada fase. No se ha realizado un tratamiento estandarizado, sino adaptado a esta persona, comenzando por los cornportamientos alimentarios y las contingencias sociales más inmediatas, para después intervenir sobre las formas de afrontar situaciones estresantes, cambios en las concepciones sobre su problemática y pensamientos asociados, y finalmente técnicas para conseguir la generalización y el mantenimiento de esos resultados en la vida diaria y frente a otros problemas futuros.

La toma de datos de línea base contínua, y en diversos parámetros de los comportamientos problemáticos, ha permitido observar la rápida solución de esos problemas, y los cambios progresivos habidos, que se mantienen un año después.

Podría argumentarse que las técnicas utilizadas han sido muy numerosas, y de todas ellas probablemente alguna funcionaría. Pero lo que se ha tratado de mostrar es el trabajo clínico diario, donde el profesional ha de tomar decisiones de tratamiento y adaptar las formas de tratamiento estandarizadas al caso individual. Se trata de mostrar la lógica de aplicación de un tratamiento multicomponente, en función de las variables determinantes que se quieren cambiar en cada fase. Los datos de línea base muestran ese cambio progresivo y su mantenimiento a largo plazo. La utilización de cada una de las técnicas tiene sentido en las variables del análisis funcional y en los objetivos clínicos perseguidos en cada fase.

El contrato de contingencias y la extinción del reforzamiento social de las "quejas psicosomáticas" es lo que funcionó rápidamente, como también ha mostrado la literatura sobre esta problemática (Fernández, 1989). Pero los problemas del individuo no terminaban en esos "síntomas". Los problemas clínicos suelen ocurrir en conjunto, con muchos problemas de pensamiento, emocionales, orientación sexual, relaciones familiares y sociales, etc., como ocurría en este caso. La evaluación inicial y el análisis funcional pueden ser, pues, las herramientas adecuadas para que el profesional vaya tomando decisiones y adaptando las técnicas estandarizadas al problema individual. 


\section{REFERENCIAS BIBLIOGRÁFICAS}

Blanchard, E.B., Schwarz, S.P., Suls, J.M., Gerardi, M.A., Scharff, L., Greene, B., Taylor, A.E., Berreman, C., y Malomood, H.S. (1992). Two controlled evaluations of multicomponent psychological treatment of irritable bowel syndrome. Behavior Research and Therapy, 30, 2, 175-189.

Fennerty, M.B. (2003). Traditional therapies for irritable bowel syndrome: An evidence-based appraisal. Review of Gastroenterology Disorders, 3, 2, 518-524.

Fernández, C. (1989). Tratamiento psicológico en el síndrome del intestino irritable. Psicothema, 1, 71-85.

González, M.C., García, E., y Fernández, C. (1992) Intervención conductual en el síndrome de intestino irritable: dos estudios clínicos. Psicothema, 4, 2, 513-530.

Greene, B., y Blanchard, E.B. (1992). Cognitive therapy for irrtable bowel synd rome. Joumal of Consulting and Clinical Psychology, 62, 3, 576-582.

Guthrie, E., Creed, F., Dawson, D., y Tomenson, B. (1992). A controled trial of psychological treatment of the irritable bowel syndrome. Gastroenterology, 102, 2, 739-740.

Heymann, I., Arnolda, R., Florinb, I, Herdab, C., Melfsenb, S., Monnikesa, $H$. (2000). The combination of medical treatment plus multicomponent behabioral therapy is superior to medical treatment alone in the therapy of irritable bowel syndrome. American Joumal of Gastroenterology, 99, 4, 981-990.

Ilnycky, A. y Bernstein, C.N. (2002) Sexual abuse in irritable bowel syndrome: to ask or not to ask, that is the question. Canadian Journal of Gastroenterology, 16, 11, 801-805.

Latimer, P.R. (1983). Functional gastrointestinal disorders. A behavioral medicine approach. New York: Springer.

Manning, A.P., Thompson, W.G, Heaton, K.W., y Morris, A.F. (1978). Toward positive diagnosis of irritable bowel syndrome. British Medical Journal, 2, 653-654.

Payne, A., y Blanchard, E.B. (1995). A controled comparison of cognitive therapy and self-help support groups in the treatment of imitable bowel syndrome. Joumal of Consulting and Clinical Psychology, 63, 5, 779-786. 
Rossouw, G.E., Stuart, A.D., y Pretorius, H.G. (2003). The role of abuse in the development of irritable bowel syndrome: A comparative study. Health Sa Gesondheid, 8, 4, 88-98.

Thompson, W.G. (1984). The irritable bowel syndrome. Gut, 25, 305320.

Walter, E.A., Katon, W.J., Roy-Byne, P.P., Jamelka, R.P., y Russo, J. (1993). Histories of sexual victimization in patients with irritable bowel syndrome or inflammatory bowel disease. American Journal of Psychiatry, 150, 10, 1502-1506.

Whitehead, W.E., Winget, C., Fedoravicius, A.S., Wooley, S. y Clackwell, B. (1982). Learned illness behavior in patients with irritable bowel syndrome and pectic ulcer. Digestive Diseases and Sciencies, 27, 202-207.

Whitehead, W.E. (1985). Psychotherapy and biofeedback in the treatment of irritable bowel syndrome. En N.W. Read (ed). Imitable Bowel Syndrome. Londres: Grune and Stratton. 


\section{Escala de valoración de síntomas y problemas}

Valore la frecuencia con que se presentan las siguientes características según esta escala:

0 - Muy baja, ninguna vez en el último mes

1 - Baja, esporádicamente, alguna vez las últimas semanas

2 - Media, dos o tres veces a la semana

3 - Alta, casi todos los días de la semana

4 - Muy alta, diariamente o varias veces al día

\begin{tabular}{|l|l|}
\hline & $0-4$ \\
\hline 1. Dolor estomacal & \\
\hline 2. Quejas sobre enfermedad & \\
\hline 3. Otras conductas de enfermedad & \\
\hline 4. Toma de medicación & \\
\hline 5. Inhibición motora (acostarse) & \\
\hline 6. Evitación de relaciones sociales & \\
\hline 7. Abandono de tareas y responsabilidades & \\
\hline 8. Dieta autoimpuesta & \\
\hline 9. Comer como antidoto & \\
\hline 10. Pautas alimentarias inadecuadas & \\
\hline 11. Conflictos familiares & \\
\hline 12. Preocupaciones & \\
\hline 13. Ansiedad / nerviosismo & \\
\hline 14. Insomnio & \\
\hline 15. Pensamientos anticipatorios & \\
\hline 16. Pensamientos distorsionados & \\
\hline 17. Pensamientos negativos & \\
\hline 18. Bajo estado de ánimo & \\
\hline 19. Baja autoestima & \\
\hline 20. Incapacidad & \\
\hline
\end{tabular}

